\title{
Erratum
}

\section{Distinct cell killing properties of the Drosophila reaper, head involution defective, and grim genes}

\author{
John P. Wing, Lei Zhou, Lawrence M. Schwartz and John R. Nambu
}

Cell Death and Differentiation, (1998) 5, 930-939

Unfortunately due to an error Figures 1,2 and 4 were reproduced as black and white instead of colour.

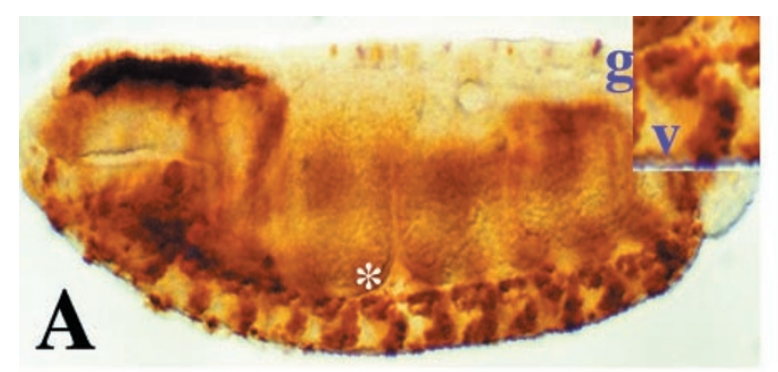

The correct versions are printed below. We apologise for any inconvenience this may have caused.
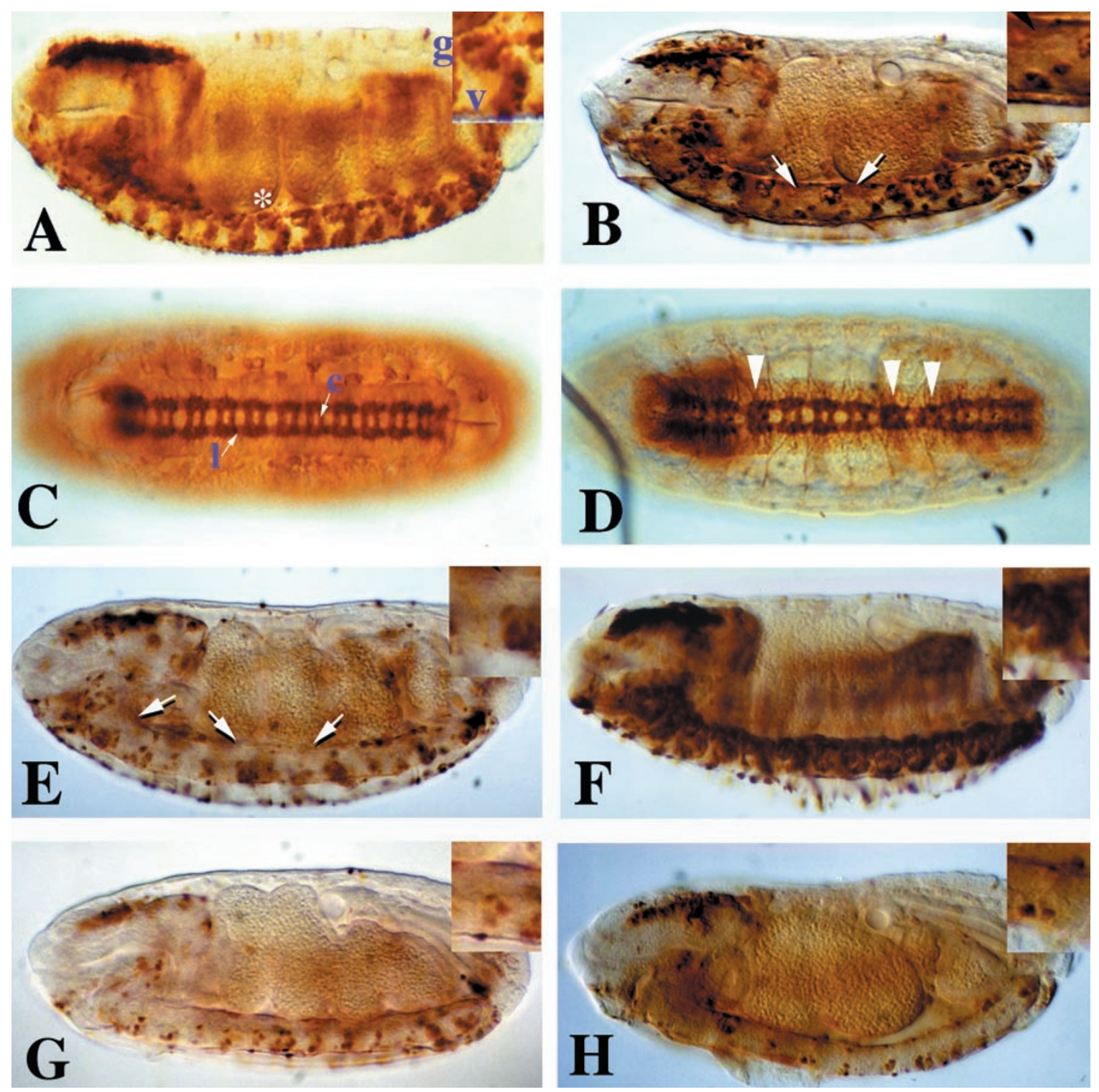

Figure 1 grim expression induces ectopic CNS midline cell death. Immunostaining of the following stage 16 embryos using either anti- $\beta$-galactosidase serum (A,B,E,F,G,H) or Mab BP102 (C,D): (A,C) P[UAS-lacZ]; P[52A-gal4]/+ (B,D) P[UAS-lacZ]; P[52A-gal4]/P[UAS-grim-2] (E) P[UAS-lacZ]; P[52A-gal4]/+; P[UASgrim-3]/+ (F) P[UAS-lacZ]; P[52A-ga/4]/P[UAS-p35]; P[UAS-grim-3]/+ (G) P[UAS-reaper]/P[UAS-lacZ]; P[52A-gal4]/P[UAS-grim-2] and (H) P[UAS-lacZ]/P[UAShid]; P[52A-gal4]/P[UAS-grim-2]. (A) Note that $\beta$-gal expression is normally detected in both midline glia at the dorsal aspect of the nerve cord and the VUM neurons at the ventral region of the nerve cord. Inset shows a higher magnification view of the A2 segment (marked by *) and indicates midline glia (g) and VUM neurons (v). (C) The axon scaffold of these embryos exhibits a wild-type organization of longitudinal connectives (1) and commissural axon bundles (c). (B) Expression of P[UAS-grim-2] typically results in the loss of midline glia from 4-5 segments of the nerve cord (arrows), and (D) results in a fused commisure phenotype (arrowheads). (E) P[UAS-grim-3] induces a more pronounced loss of midline glia (arrows) as well as VUM neurons. (F) grim-induced ectopic midline cell death is blocked by co-expression of the baculovirus p35 protein. $(\mathbf{G}, \mathbf{H})$ Co-expression of grim and reaper $(\mathbf{G})$ or grim and hid $(\mathbf{H})$ result in a complete elimination of the midline glia as well as partial elimination of the VUM neurons. Compare this to the moderate midline cell death in (B), and absence of midline cell death induced by expression of reaper or hid alone (Zhou et al, 1997). All views are sagittal with anterior to left and dorsal up 

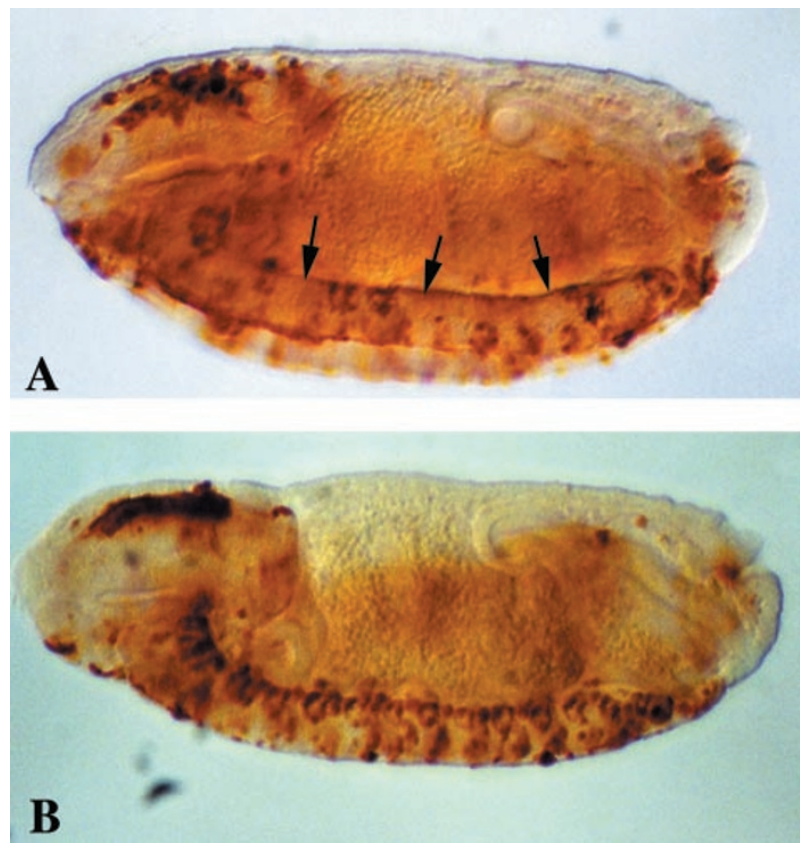

Figure 2 diap2 does not block grim-induced CNS midline cell death. Anti- $\beta$ galactosidase staining of the following stage 16 embryos: (A) P[UAS-lacZ; $\mathrm{P}[52 \mathrm{~A}-$ gal4]/P[UAS-grim]; P[UAS-diap2]/+ and (B) P[UAS-lacZ]/P[UASreaper]-P[UAS-hid]; P[52A-gal4]/+; P[UAS-diap2]/+. Note that diap2 fails to block grim-induced midline cell death $(\mathbf{A})$, as several segments still lack midline glia (arrows). diap2 does block midline cell deaths induced by coexpression of reaper and hid, (B), as wild-type numbers of cells are detected. All views are sagittal with anterior to left and dorsal up
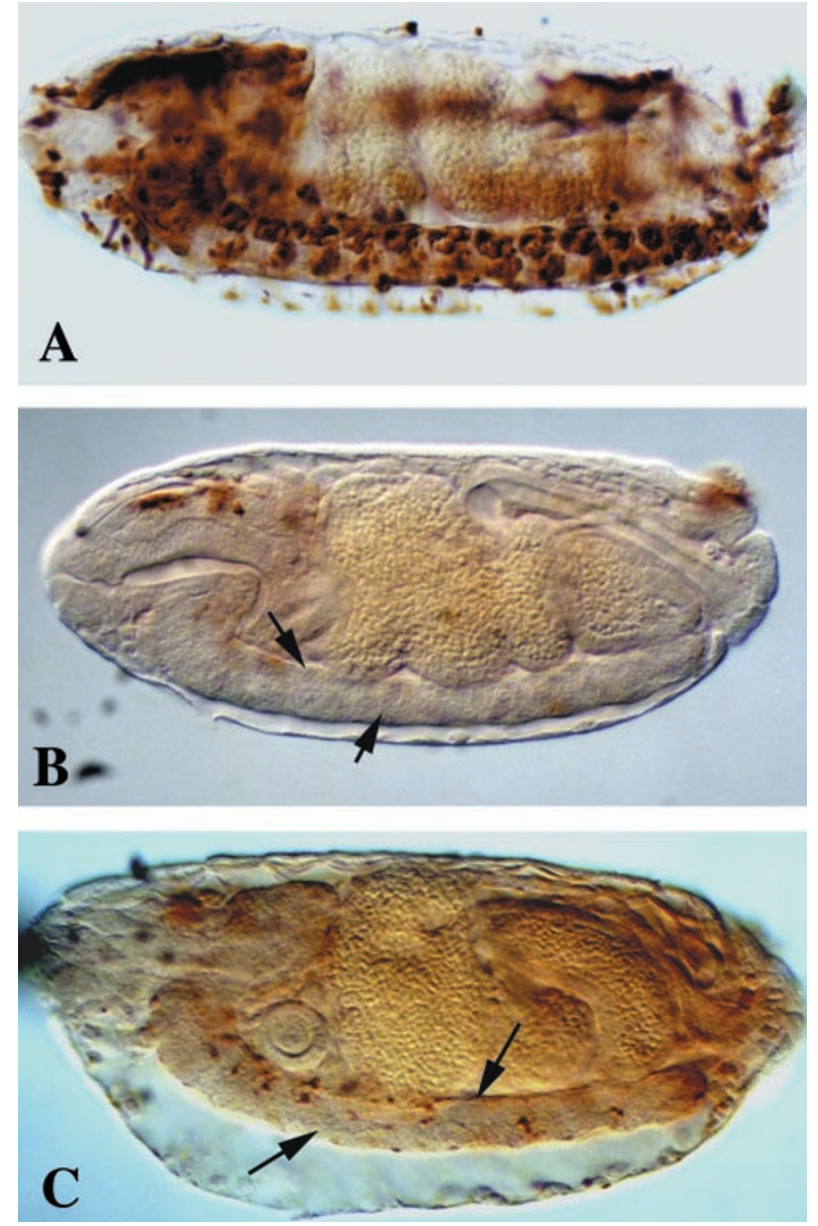

Figure 4 A Reaper-C protein lacking the conserved RHG domain can act cooperatively with Hid and Grim to induce CNS midline cell death. (A-C) Anti- $\beta$ galactosidase staining of the following stage 16 embryos: (A)P[UAS-lacZ]; $\mathrm{P}[52 \mathrm{~A}$-gal4]/+; P[UAS-reaper-C-3]/+ (B) P[UAS-lacZ]; P[52A-gal4]/P[UASgrim-2]; P[UAS-reaper-C-3]/+ and (C) P[UAS-lacZ/P[UAS-hid]/; P[52A-gal4]/ +; P[UAS-reaper-C-3]/+. Note that Reaper-C expression alone does not result in any ectopic midline cell death (A). Co-expression of Reaper-C with Grim (B) or Hid (C) results in severe loss of both midline glia and VUM neurons (arrows). This phenotype is more severe than that seen when full length Reaper is expressed with Grim (compare to Figure 1G) or Hid (see Zhou et al, 1997). All views are sagittal with anterior to left and dorsal up 